\title{
Luminance-dependent hue shift in protanopes
}

\author{
DAVID L. BIMLER ${ }^{1}$ AND GALINA V. PARAMEI ${ }^{2}$ \\ ${ }^{1}$ Department of Health and Human Development, Massey University, New Zealand \\ ${ }^{2}$ Institute of Medical Psychology, Otto-von-Guericke University of Magdeburg, Germany
}

(Received September 7, 2003; AcCEPTED January 16, 2004)

\begin{abstract}
For normal trichromats, the hue of a light can change as its luminance varies. This Bezold-Brücke (B-B) hue shift is commonly attributed to nonlinearity in the blue-yellow opponent system. In the present study, we questioned whether protanopes experience analogous changes. Two protanopes (Ps) viewed spectral lights at six luminance levels across three log steps. Two normal trichromats (NTs) were tested for comparison. A variant of the color-naming method was used, with an additional "white" term. To overcome the difficulty of Ps' idiosyncratic color naming, we converted color-naming functions into individual color spaces, by way of interstimulus similarities and multidimensional scaling (MDS). The color spaces describe each stimulus in terms of spatial coordinates, so that hue shifts are measured geometrically, as displacements along specific dimensions. For the NTs, a B-B shift derived through MDS agreed well with values obtained directly by matching color-naming functions. A change in color appearance was also observed for the Ps, distinct from that in perceived brightness. This change was about twice as large as the B-B shift for NTs and combined what the latter would distinguish as hue and saturation shifts. The protanopic analogue of the B-B shift indicates that the blue-yellow nonlinearity persists in the absence of a red-green signal. In addition, at mesopic levels ( $\leq 38 \mathrm{td}$ ), the Ps' MDS solution was two dimensional at longer wavelengths, suggesting rod input. Conversely, at higher luminance levels (76 td-760 td) the MDS solution was essentially one dimensional, placing a lower limit on S-cone input at longer wavelengths.
\end{abstract}

Keywords: Bezold-Brücke hue shift, Protanope, Color naming, Geometrical model, Nonlinearity

\section{Introduction}

Increasing luminance can alter the hue and saturation of a light, as perceived by a normal trichromat, or NT (Purdy, 1937). Hue changes are known as the Bezold-Brücke (B-B) shift and commonly attributed to nonlinearity in the blue-yellow opponent system (e.g., Larimer et al., 1975; Werner \& Wooten, 1979; but also see Hurvich, 1981, pp. 73-74). Here we examine whether protanopic observers (Ps) experience an analogous shift in the absence of a red-green opponent dimension. The behavior of a B-B shift in Ps can potentially shed light on nonlinear transformations in the color-processing system.

To estimate color appearance in NTs at varying luminances, the method of color naming has been used (Boynton \& Gordon, 1965). To quantify the B-B shift, color-naming functions are matched across luminance levels. To elicit a description of (for instance) $80 \%$ "yellow" and 20\% "red" from an observer might require chromatic stimuli of $580 \mathrm{~nm}$ at low and $590 \mathrm{~nm}$ at high luminance (the longer wavelengths becoming subjectively yellower as luminance increases).

The reliability of the color-naming procedure for Ps has been established (Scheibner \& Boynton, 1968). However, the luminance-

Address correspondence and reprint requests to: Galina V. Paramei, Institute of Medical Psychology, Otto-von-Guericke University of Magdeburg, Leipziger Strasse 44, 39120 Magdeburg, Germany. E-mail: galina.paramei@medizin.uni-magdeburg.de matching analysis of their data is problematic, because there may be no combination of color terms at high luminance that matches the specific combination a dichromat uses to describe a lowluminance stimulus. Dichromats may fail to disentangle luminance and hue in their use of terms like "green" or "yellow" (Paramei \& Cavonius, 1997), while the mutual exclusivity of "red" and "green", or "blue" and "yellow" is not assured.

One way of overcoming the problem uses multidimensional scaling, or MDS (Shepard \& Carroll, 1966; Gordon \& Abramov, 1988). The outcome is a geometrical model in which points represent stimuli, so each stimulus is specified by a spatial location rather than by color-name combination, while hue shifts are measured as luminance-dependent displacements of those locations. Points are arranged so that distances between them reflect the similarities between corresponding pairs of stimuli; similarities in turn are derived by comparing color-name profiles.

In the present study, this geometric approach is used to quantify the shifting subjective properties of chromatic stimuli for Ps while varying luminance across three log steps-from mesopic, through low photopic, to high photopic levels. For validation, similar data from NTs are analyzed in the same way, and by matching luminances.

\section{Materials and methods}

The experiment was conducted at Moscow State University. Subjects were dizygote twin males aged 23 years, AA and DA, 
diagnosed as Ps using Rabkin pseudoisochromatic plates (Rabkin, 1971). On an anomaloscope (Rautian, 1957) they accepted the full range of Rayleigh-equation matches. NT subjects were two unrelated females, aged 25 and 30 years. All subjects were native Russian speakers. The experiment was conducted in accordance with principles embodied in the Declaration of Helsinki (Code of Ethics of the World Medical Association).

Monochromatic stimuli and a broadband white stimulus were produced by passing light from a KGM-24 tungsten filament source $(c a .2850 \mathrm{~K})$ through Zeiss interference filters, and a neutral filter, respectively (Izmailov \& Sokolov, 1991, Fig. 1). NTs viewed 20 monochromatic stimuli at approximately equal steps along the spectrum from 405 to $675 \mathrm{~nm}$. For the Ps, five more filters were added around $500 \mathrm{~nm}$, in the region of the protanopic achromatic point. Six luminance levels were used: 200, 100, 20, 10,2 , and $0.2 \mathrm{~cd} / \mathrm{m}^{2}(760,380,76,38,7.6$, and $0.76 \mathrm{td})$, with luminance measured by a VFM-57 photometer and controlled with a neutral-density wedge. Thus, $6 \times 21=126$ stimuli were presented to NTs, and $6 \times 26=156$ to Ps.

Stimuli were presented foveally in Maxwellian view. A 2.2-deg circular test field was exposed against a dark surround for $3 \mathrm{~s}$ followed by $15-20 \mathrm{~s}$ of darkness. Subjects were dark adapted for 20 min prior to the session. The NTs' right eyes were tested, while both eyes were tested for Ps.

Observers judged each stimulus using five response categories: the Russian equivalents of red (R), yellow (Y), green $(\mathrm{G})$, and blue (B) to describe the hue, and white (W) for the achromatic content. Siniy, the term used for B, is the closest equivalent (Abramov et al., 1997). One, two, or three terms could be used, in order of salience. Ten points were shared amongst those terms (if two terms were used, the first was given 6 and the second 4; three terms received 5, 3, and 2 points). Each combination of wavelength and luminance was presented 20 times over ten sessions, twice per session, in pseudorandom order. Summed over presentations, the points for each color name gave the color-naming function. This is an extension of previously reported data (Paramei et al., 1998).

The two NTs' data were sufficiently similar to be averaged and written as a $126 \times 5$ matrix. Averaging data from both eyes of both Ps gave a $156 \times 5$ matrix. Next, the similarity between any pair of stimuli was quantified as the Pearson correlation between the corresponding rows (Gordon \& Abramov, 1988), yielding $126 \times$ 126 and $156 \times 156$ similarity matrices. These were analyzed with a nonmetric MDS algorithm (Kruskal, 1964) to provide geometric representations.

\section{Results and discussion}

\section{Hue shifts in color spaces for NTs}

MDS models with two, three, and four dimensions had Stress values (i.e., badness-of-fit) of $0.15,0.09$, and 0.07 , respectively. As a substantial improvement over two dimensions, three dimensions were optimal, representing the $i$ th stimulus by a point $x_{i}$ with coordinates $x_{i 1}, x_{i 2}, x_{i 3}$. This model was rotated to maximize the association between the $x_{i 3}$ (D3 coordinates) and the W-naming score for the corresponding stimuli. D3 was thus an achromatic or "desaturation" axis, while $D 1$ and $D 2$ were R-G and B-Y axes. NTs seemingly did not use the available color vocabulary to describe luminance, since no correlate could be found in the solution for that aspect of stimuli.

The position of the points $\boldsymbol{x}_{i}$ were expressed as spherical coordinates: azimuth $\Theta_{i}=\arctan \left(x_{i 2} / x_{i 1}\right)$; altitude $\Psi_{i}=\arctan$

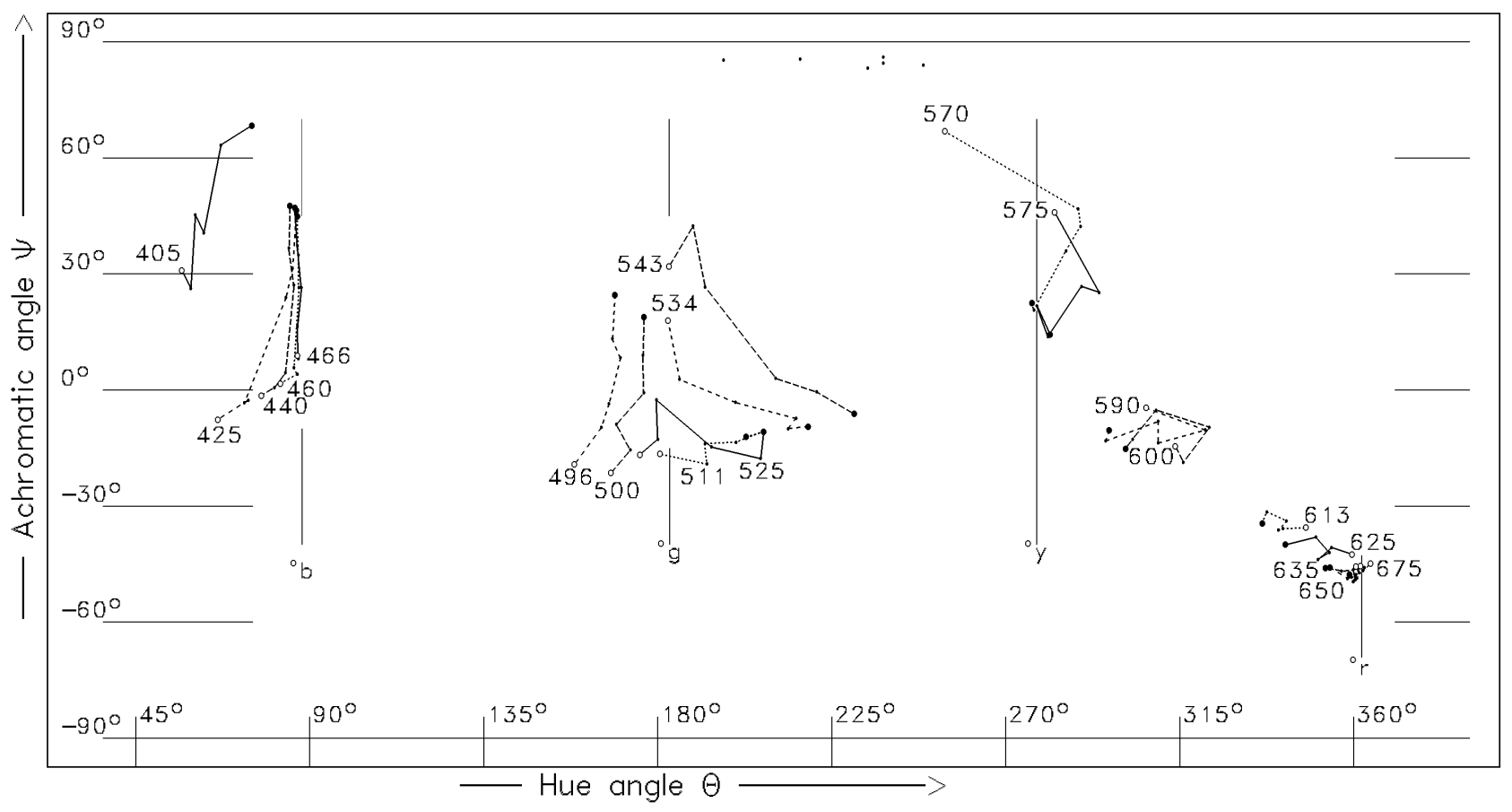

Fig. 1. Color space for normal trichromats, plotted in spherical coordinates. Horizontal axis $=$ hue angle $\Theta$. Vertical axis $=$ achromatic angle $\Psi$ (desaturation). Same-wavelength stimuli linked in order of increasing luminance, from 0.76 td (open circles) to 760 td (solid circles). Six unlabelled points near $\Psi=90 \mathrm{deg}$ (i.e., at the positive extreme of $D 3$ ) represent the $\mathrm{W}$ stimuli. Hue angles where stimuli are "unique hues" ${ }^{\circ} \mathrm{b},{ }^{\circ} \mathrm{g},{ }^{\circ} \mathrm{y},{ }^{\circ} \mathrm{r}$ in the raw data are marked as vertical lines. 
$\left[x_{i 3} /\left(x_{i 1}^{2}+x_{i 2}^{2}\right)^{1 / 2}\right]$. We identify $\Theta$ and $\Psi$ as hue and achromatic angle, respectively. Points also have a radial coordinate $r_{i}$, that is, distance from the origin, but the standard deviation of $r_{i}$ was only $4.1 \%$ of the mean value. $\Theta_{i}$ and $\Psi_{i}$ are plotted in Fig. 1. Fig. 1 also shows four "unique hue" loci, that is, values of $\Theta$ where stimuli are located that the observers described with a single opponent-hue name (e.g., as G, with no component of B or Y).

Fig. 1 shows that chromatic stimuli vary substantially in $\Psi(\lambda$, $L$ ), that is, W-naming. The lowest values of $\Psi$ (highest saturation) appear at long wavelengths (described as R) and around $511 \mathrm{~nm}$ (where G-naming peaks). Increasing luminance has the effect of decreasing saturation for $\lambda<511 \mathrm{~nm}$, while increasing it for longer wavelengths (particularly for stimuli described as yellow).

The horizontal and vertical displacement between samewavelength points distinguish two effects of luminance change, on hue and saturation, respectively. For two luminance levels, $L_{1}, L_{2}$, the B-B shift at wavelength $\lambda_{1}$ is $\Delta \lambda=\lambda_{2}-\lambda_{1}$, where $\lambda_{2}$ is found by matching hue angles (interpolating if necessary), that is, $\Theta\left(\lambda_{1}, L_{1}\right)=\Theta\left(\lambda_{2}, L_{2}\right) . \Delta \lambda$ is plotted in Fig. 2, in which the highest level of $L$ is compared with each of the lower levels. The hue shift peaks in the green-yellow region at $535 \mathrm{~nm}$ for the smallest luminance difference and at $511 \mathrm{~nm}$ for larger differences. The values are well in accord with those obtained by direct measures in previous studies (Purdy, 1937; Boynton \& Gordon, 1965; Gordon \& Abramov, 1988), though the lack of blue-green stimuli around $500 \mathrm{~nm}$ prevents us from estimating the shift in that spectral region.

Chromatic shifts in color spaces for Ps

Averaged data are presented here, though it is worth noting that individual solutions for DA and AA differed in specifics because differences in their use of color terms led to loss of information in particular spectral regions. A three-dimensional MDS solution was optimal for the averaged color-naming data from the Ps, with Stress $_{1}=0.13$ (compared to values of 0.19 and 0.11 for two and four dimensions). This is compatible with the reduced dimensionality of dichromatic vision, because the Ps evidently used the available terms to describe variations in stimulus intensity: luminance emerged as a third dimension (D3). Retaining three dimensions also avoided artefacts that emerged in a two-dimensional solution, and eased comparisons with the solution for the NTs.

The solution is locally two dimensional, with points $\boldsymbol{x}_{i}$ lying close to a spherical surface: the distances $r_{i}$ from the $\boldsymbol{x}_{i}$ to the origin were distributed with a standard deviation that was $5.7 \%$ of mean $r_{i}$. As with NTs, locations on this spherical surface can be

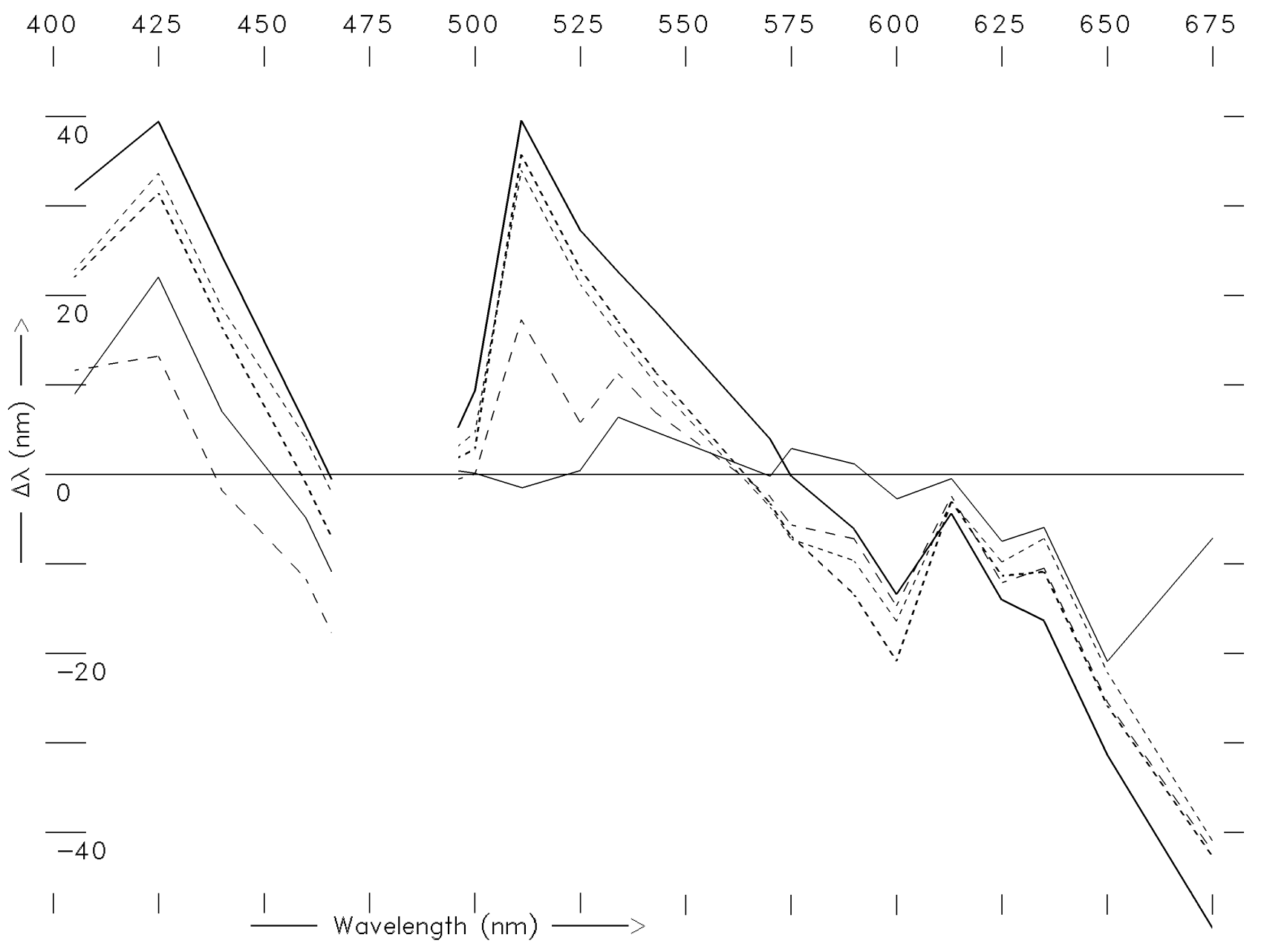

Fig. 2. Bezold-Brücke hue shift in normal trichromats, comparing 760 td stimuli against lower luminance values, from 380 td (— to $0.76 \mathrm{td}(-)$. Horizontal axis $=\lambda$. Vertical axis $=\Delta \lambda$, the change in wavelength required to compensate for a difference in luminance and produce the same hue angle. 
expressed as angular coordinates $\Theta$ and $\Psi$ (Fig. 3). In a departure from the NT case, the altitude $\Psi$ (and D3) separates the six luminance levels (specifically separating the six luminances of White in the vicinity of the neutral 511-nm stimuli). We interpret $\Psi$ as a perceived brightness coordinate. As expected, perceived brightness is reduced at longer wavelengths where the protanopes' retinal efficiency declines.

The azimuth angle $\Theta$ provides some separation of stimuli by wavelength, and can again be interpreted as "hue angle". This is despite considerable overlap between the protanopes' $\mathrm{R}$ - and G-naming functions (not shown for reasons of space). This precludes any interpretation of $D 1$ and $D 2$ in terms of opponent hues, and they are merely mathematical conveniences. Thus the starting point for $\Theta(0 \mathrm{deg}=360 \mathrm{deg})$ is arbitrary. Inspection of the raw color-naming functions revealed no element of B-naming in the long-wavelength responses, nor of R-naming at short wavelengths, so $\Theta$ ranges around (slightly) less of the sphere than in the NT case.

Note that $\Theta$ is an index of saturation as well as hue. Saturation is zero at the neutral point $(\lambda \sim 511 \mathrm{~nm}, \Theta \sim 200 \mathrm{deg})$; it increases with $\Theta$ for longer wavelengths, but also increases with decreasing $\Theta$ for shorter wavelengths. Thus, if protanopes possess any analogue to the luminance/saturation effect in NTs, one would expect the desaturation of high-luminance, short-wavelength stimuli to displace them longitudinally towards the neutral point. Conversely, the saturation of high-luminance, long-wavelength stimuli should sweep them away from the neutral point.

Luminance-induced hue/saturation shifts $\Delta \lambda$ were calculated by matching hue angles $\Theta$, and plotted in Fig. 4 . The values are quite similar in profile to those obtained from NTs but about double the magnitude. The general picture is consistent with the protanope observers experiencing an equivalent to the B-B shift, but overlaid with what in NTs is a separate shift, in stimulus saturation.

The MDS solution for protanopes could be rotated to different axes. This would affect the specific values of $\Delta \lambda$, but not the essential feature of the solution-which is the relative displacement of points representing high-luminance stimuli, compared to low-luminance points.

If M-cones alone subserved protanopic perception of the longwavelength end of the spectrum, this region should collapse into one dimension-with perceived brightness as the only quality distinguishing the stimuli. Such pattern is indeed observed in Fig. 3, for stimuli in the range 76-760 td (the three higher luminance levels here) and $\lambda>600 \mathrm{~nm}$. For example, 635-nm stimuli of varying luminance are arranged in sequence of perceived brightness, but $675-\mathrm{nm}$ stimuli fall along the same sequence, displaced along it by lower retinal efficiency at the longer wavelength. Thus, the present results complement earlier findings (McMahon \& MacLeod, 1998) that S-cones contribute to discrimination at sufficiently high intensities $\left(\sim 10^{4} \mathrm{td}\right)$. Significant $\mathrm{S}$-cone input at longer wavelengths would displace stimuli away from the sequence in Fig. 3; the absence of any displacement places a lower bound upon the required intensity.

In contrast, low-luminance stimuli ( $L \leq 38$ td) occupy a second dimension even at long wavelengths, that is, the Ps distinguished them other than by perceived brightness. It is possible that rod signals feed into chromatic pathways at mesopic light levels and contribute to protanopic chromatic sensation (Buck et al., 2000).

\section{Conclusion}

In protanopes, luminance-dependent changes in the appearance of spectral stimuli show the features that would be predicted by

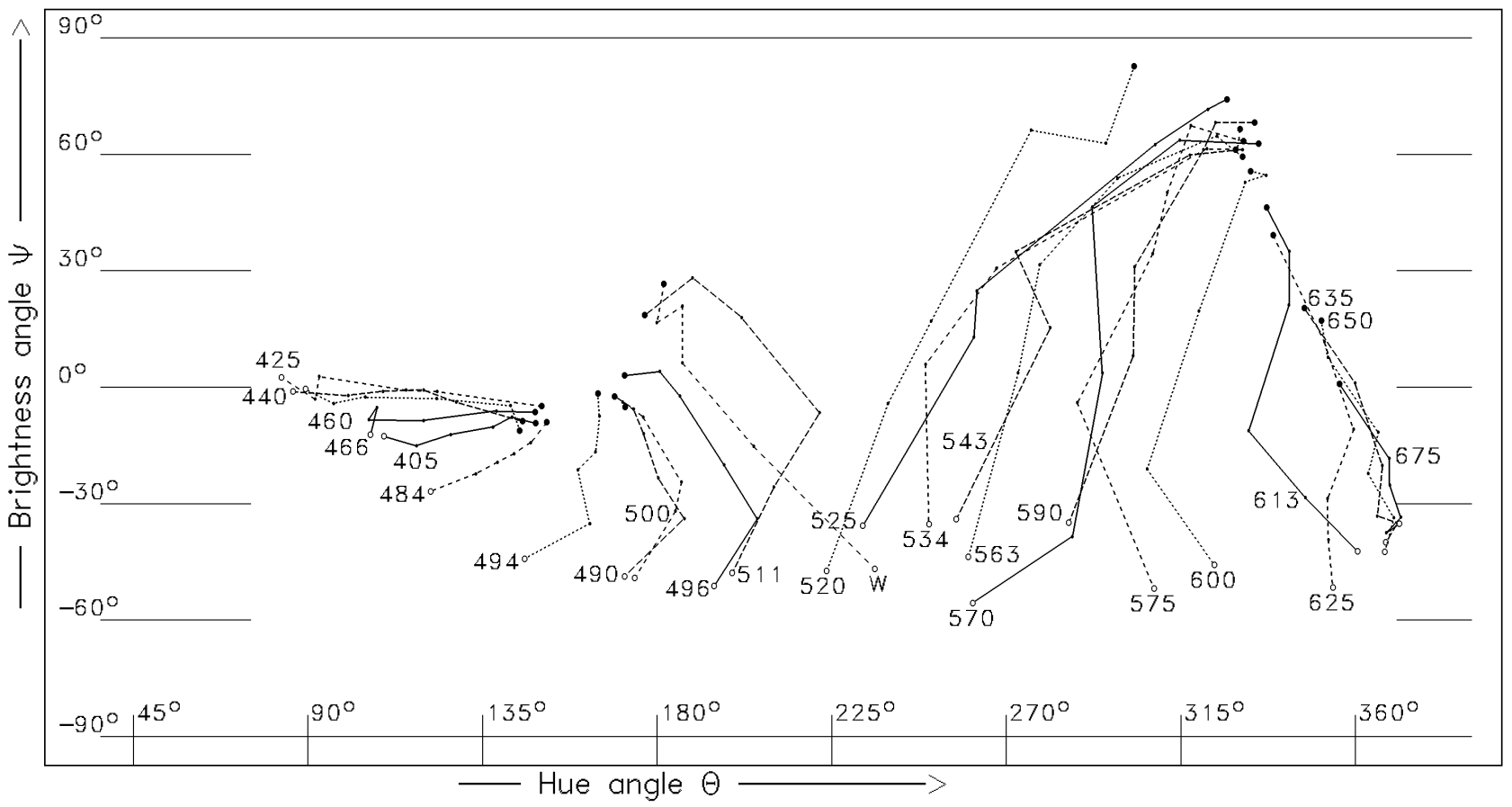

Fig. 3. Color space for protanopes, plotted in spherical coordinates. Horizontal axis $=$ hue/saturation angle $\Theta$. Vertical axis $=$ brightness angle $\Psi$. Same-wavelength stimuli linked in order of increasing luminance, from $0.76 \mathrm{td}$ (open circles) to 760 td (solid circles). 


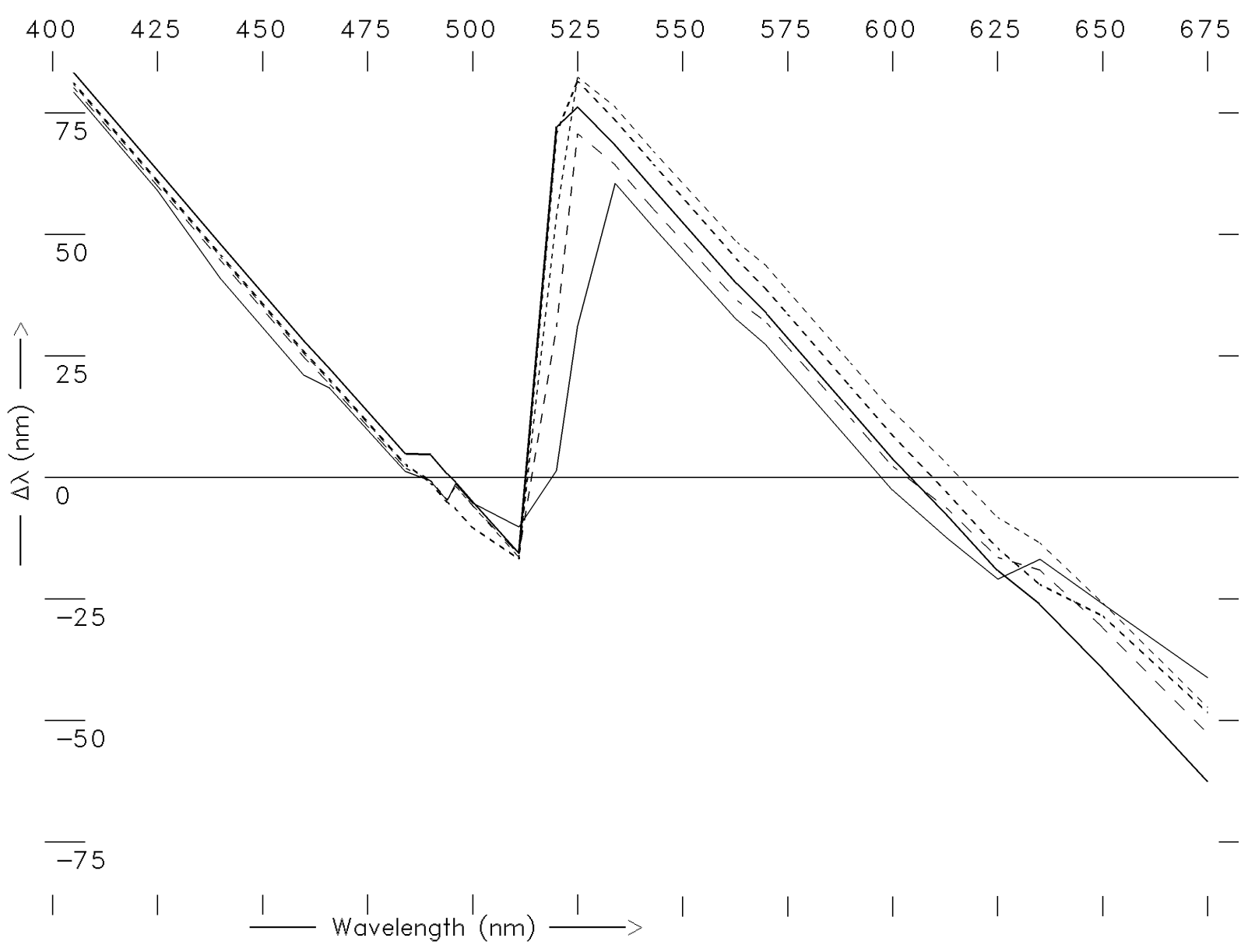

Fig. 4. An analogue of the Bezold-Brücke hue shift for protanopes, comparing 760-td stimuli against lower luminance values from 380 td (-) to $0.76 \mathrm{td}$ (—). Axes as in Fig. 2 (with different scale on the vertical axis).

superimposing the shifts in hue and saturation observed in normal trichromats. The protanopic analogue of the Bezold-Brücke shift indicates that the blue-yellow nonlinearity persists in the absence of a red-green signal (presumably disrupted in these observers by the absence of L photoreceptors).

\section{Acknowledgments}

The authors are grateful for two anonymous reviewers of an earlier version of the manuscript for valuable comments and criticisms, and to Chingis A. Izmailov for his help in retrieving technical specifications.

\section{References}

Abramov, I., Gordon, J., Akilov, V., Babiy, M., Bakis, G., Ilyusha, S., Khamermesh, K. \& VAYNer, A. (1997). Color appearance: Singing the Russian blues. Investigative Ophthalmology and Visual Science 38, S899.

Boynton, R.M. \& GoRdon, J. (1965). Bezold-Brücke hue shift measured by color-naming technique. Journal of the Optical Society of America $\mathbf{5 5}, 78-86$.

Buck, S.L., Knight, R. \& Bechtold, J. (2000). Opponent-color models and the influence of rod signals on the loci of unique hues. Vision Research 40, 3333-3344.

Gordon, J. \& Abramov, I. (1988). Scaling procedures for specifying color appearance. Color Research and Application 13, 146-152.

Hurvich, L.M. (1981). Color Vision. Sunderland, Massachusetts: Sinauer Associates.
Izmailov, Ch.A. \& Sokolov, E.N. (1991). Spherical model of color and brightness discrimination. Psychological Science 2, 249-259.

KrusKal, J.B. (1964). Multidimensional scaling by optimizing goodness of fit to a nonmetric hypothesis. Psychometrika 28, 1-27.

Larimer, J., Krantz, D.H. \& Cicerone, C.M. (1975). Opponent-process additivity: II. Yellow/blue equilibria and nonlinear models. Vision Research 15, 723-731.

McMahon, M.J. \& MAcLeod, D.I.A. (1998). Dichromatic color vision at very high light levels: Red/green discrimination using the bluesensitive mechanisms. Vision Research 38, 973-983.

Paramei, G.V., Bimler, D.L. \& Cavonius, C.R. (1998). Effects of luminance on color perception of protanopes. Vision Research 38, 3397-3401.

Paramei, G.V. \& Cavonius, C.R. (1997). Color naming in dizygotic twin protanopes at different luminance levels. In AIC Color 97. Proceedings of the 8th Congress of the International Colour Association, Vol. I, pp. 335-338. Kyoto, Japan: The Color Science Association of Japan.

PuRDY, D.M. (1937). The Bezold-Brücke phenomenon and contours for constant hue. American Journal of Psychology 49, 313-315.

RABKIN E.B. (1971). [Pseudoisochromatic Plates for diagnostics of color sensitivity] (in Russian). Moscow: Meditsina.

Rautian, G.N. (1957). [A new anomaloscope] (in Russian). Biofizika 2, $734-742$.

Scheibner, H.M.O. \& Boynton, R.M. (1968). Residual red-green discrimination in dichromats. Journal of the Optical Society of America 58, 1151-1158.

ShePard, R.N. \& Carroll, J.D. (1966). Parametric representation of nonlinear data structures. In Multivariate Analysis, ed. KrishnaiaH, P.R., pp. 561-592. New York: Academic Press.

WERNER, J.S. \& WoOTEN, B.R. (1979). Opponent chromatic mechanisms: Relation to photopigments and hue naming. Journal of the Optical Society of America 69, 422-434. 


\title{
Luminance-dependent hue shift in protanopes
}

\author{
Bimler, David L.
}

2004

http://hdl.handle.net/10179/9668

22/04/2023 - Downloaded from MASSEY RESEARCH ONLINE 